\title{
DOSES REDUZIDAS DE HERBICIDAS DE PÓS-EMERGÊNCIA PARA CONTROLE DE PAPUA EM SOJA $^{1}$
}

\author{
NILSON G. FLECK ${ }^{2}$
}

\begin{abstract}
Em 1992/93 foram conduzidos dois experimentos a campo em Eldorado do Sul, RS, objetivando avaliar os herbicidas haloxyfop e sethoxydim no controle de papuã (Brachiaria plantaginea) em doses mais reduzidas que as usuais. Haloxyfop foi testado nas doses de 120, 90, 60, 30 e $30+30 \mathrm{~g} / \mathrm{ha}$. Sethoxydim foi avaliado nas doses de 184,138 , 92,46 e $46+46 \mathrm{~g} / \mathrm{ha}$. Os herbicidas foram aspergidos 7 e 14 dias após semeadura da soja nos experimentos 1 e 2 quando as plantas de papuã encontravam-se com 1 a 2 folhas e com 3 a 4 folhas, respectivamente. As doses plenas foram aspergidas 2 semanas mais tarde que as doses reduzidas. Foi possível obter nível aceitável de controle de papuã em alguns tratamentos, em função do produto, da época e do método de aplicação. Aplicações seqüienciais de doses reduzidas de
\end{abstract}

RESUMO haloxyfop $(30+30 \mathrm{~g} / \mathrm{ha})$ alcançaram controle de 72 e $95 \%$, médias dos experimentos 1 e 2 , respectivamente. No experimento 1, níveis de produtivídade de soja equivalentes ao do tratamento capinado só foram obtidos com doses plenas de haloxyfop e sethoxydim. No experimento 2 , produtividades equivalentes à do capinado foram alcançadas nas doses plenas dos dois herbicidas e ainda com haloxyfop a $90 \mathrm{~g} / \mathrm{ha}$ e a $30+30 \mathrm{~g} / \mathrm{ha}$. A pesquisa evidencia a necessidade do papuã ser totalmente eliminado para ser atingido rendimento $m a ́$ ximo de soja.

Palavras-chave: Aplicações seqüênciais, doses fracionadas, haloxyfop, sethoxydim, interferência de papuã, Brachiaria plantaginea, Glycine max.

\section{ABSTRACT \\ Reduced rates of post-emergence herbicides for alexandergrass control in soybean}

Two trials were conducted under field conditions during the 1992/93 growing season in Eldorado do Sul, RS, Brazil, in order to evaluate haloxyfop and sethoxydim herbicides for Alexandergrass (Brachiaria plantaginea) control using rates lower than the usual. Haloxyfop rates tested were $120,90,60,30$, and $30+30 \mathrm{~g} / \mathrm{ha}$. Sethoxydim was evaluated under rates of 184, 138, 92, 46, and $46+46 \mathrm{~g} / \mathrm{ha}$. Herbicides were sprayed 7 and 14 days after soybean seeding date for experiments 1 and 2, when Alexandergrass plants presented 1 to 2 and 3 to 4 leaves, respectively. Herbicide full rates were applied 2 weeks after the reduced ones. It was possible to attain acceptable level of Alexandergrass control only with a few treatments, these being a function of the compound used, and also of time and method of application.
Sequencial applications of reduced rates of haloxyfop $(30+$ $30 \mathrm{~g} / \mathrm{ha}$ ) achieved control levels of 72 and $95 \%$, as averages for experiments 1 and 2, respectively. For experiment 1 , soybean yield levels equivalent to hand hoeing were attained only by the full rates of haloxyfop and sethoxydim. In experiment 2, yields equivalent to the hoeing treatment were achíeved by full rates of both products and also by haloxyfop at $90 \mathrm{~g} / \mathrm{ha}$ and sequencial applications of $30+30 \mathrm{~g} / \mathrm{ha}$. The research also attest the necessity of complete elimination of Alexandergrass infestation in order to get maximum soybean yield.

Additional index words: Sequencial applications, split rates, haloxyfop, sethoxydim, signalgrass interference, Brachiaria plantaginea, Glycine max.

\footnotetext{
${ }^{1}$ Recebido para publicação em 06/10/93 e na forma revisada em 21/12/93. Trabalho financiado por CNPq, FAPERS e PROPESPIUFRS

${ }^{2}$ Eng $^{\circ}$ Agr $^{\circ}$, PhD, Prof. Adjunto, Bolsista do CNPq, Dep. Plantas de Lavoura, Fac. de Agronomia, UFRS. Cx. Postal 776, CEP 90001-000, P. Alegre, RS
} 


\section{INTRODUÇÃO}

Os prejuízos econômicos causados à produção de soja pela interferência de plantas daninhas, os decréscimos na qualidade dos grãos colhidos e as reduções na eficiência da operação de colheita devido à presença de plantas daninhas tem sido investigados e comprovados por diversos pesquisadores. As plantas daninhas causam também perdas econômicas na produção de soja devido ao custo dos herbicidas e dos métodos culturais e mecânicos de controle que devem ser utilizados. Os herbicidas tornaram-se o método mais importante para controle de plantas daninhas em soja nas últimas décadas (DeFelíce et a!., 1989). Diversos herbicidas seletivos de pós-emergência entraram no mercado para controle de plantas daninhas em soja durante a metade da década de 70 e durante a de 80 (Steckel et al., 1990; Klingaman et al., 1992). Esses produtos têm sido usados com sucesso durante muitos anos (Devlin et a!., 1991). As aplicações de pósemergência apresentam a vantagem sobre aplicações ao solo, no sentido de que podem ser usadas após a emergência e permitem a precisa identificação das ervas. Dessa forma, torna-se possível recomendar o herbicida correto para as espécies presentes.

Os baixos preços da soja no final da década de 70 e início da de 80 levaram os agricultores a buscar maneiras de reduzir os custos de produção (Steckel et al., 1990). A contaminação da água subterrânea com agroquímicos tornou-se também uma grande preocupação mundial nesta época. Em resposta aos baixos preços dos grãos e ao aumento das preocupações com o ambiente, os pesquisadores passaram a desenvolver práticas de controle das plantas daninhas que trouxes sem vantagens de custo e segurança ecológica (Prots ko \& Meade, 1993). Ocorreu, então, uma mudança para o emprego do cultivo conservacionista em muitas áreas, e as preocupações sobre resíduos de agroquímicos no ambiente encorajaram pesquisadores a investigar maneiras de controlar plantas daninhas com redução do uso de herbicidas (DeFelice et al., 1989).

Os métodos propostos para reduzir os custos de produção e a quantidade de agroquímicos no ambiente incluíram a integração das opções de manejo, como aumento do uso do cultivo ou capina, e o emprego de doses de herbicidas abaixo das referidas nos rótulos dos produtos (Steckel et al., 1990). Maneiras óbvias de reduzir o custo do controle de plantas daninhas são diminuir o número de aplicações herbicidas, realizar aplicações em áreas mais restritas como em faixas, e/ou reduzir as doses herbicidas requeridas para alcançar controle aceitável das mesmas (DeFelice et al., 1989). Uma técnica de manejo que, potencialmente, satisfaz tais necessidades é o uso de doses reduzidas de herbicidas. Esta técnica apresenta o potencial de aumentar os lucros dos agricultores (Devlin et a!., 1991; Prostko \& Meade, 1993). Utilizar doses reduzidas de herbicidas de pós-emergência pode representar, além de potencial redução do custo do controle (Wallace \& Bellinder, 1990; Carey et al., 1992), a limitação da quantidade de agroquímicos introduzidos no ambiente, minimizando a exposição do solo e a contaminação da água superficial ou subterrânea por tais produtos (Steckel et al., 1990; Devlin et a!., 1991; Griffin et al., 1992; Prostko \& Meade, 1993). Existe igualmente potencial na redução das doses de herbici das de pré-emergência (Wallace \& Bellinder, 1990). O uso de doses reduzidas minimiza também os efeitos potenciais da persistência desses produtos no solo e suas conseqüencias sobre culturas subseqüentes sensíveis, participantes de sistemas de rotação (Klinga man et al., 1992).

As doses de herbicidas normalmente recomendadas nos rótulos são estabelecidas pelos fabricantes em níveis elevados para assegurar o controle eficiente das espécies sobre uma ampla faixa de condições de manejo e de ambiente (Devlin et a!., 1991). A dose de herbicida necessária para obter controle eficiente em pós-emergência é dependente das ervas-alvo, da idade ou estádio de crescimento das mesmas e das condições ambientais na época da aplicação herbicida (King \& Oliver, 1992). Assim, as doses de rótulo estão ajustadas para assegurar controle de uma faixa de numerosas espécies, que variam em suscetibilidade sob condições que podem estar abaixo daquelas consideradas ótimas para exercerem plena atividade herbicida (King \& Oliver, 1992; Klingaman et al., 1992). Em certas situações, adotando-se manejo adequado e/ou sob determinadas condições de ambiente, as doses de herbicidas podem ser reduzidas e ainda assim controlar eficientemente as plantas daninhas (Devlin et al., 1991).

Herbicidas de pós-emergência aplicados em doses abaixo das recomendadas podem, efetivamente, controlar espécies suscetíveis de ervas, quando utilizados em plantas jovens e sob condições ambientais favoráveis, de modo a promover seu crescimento ativo (Devlin et al., 1991; Griffin et al., 1992; King \& Oliver, 1992; Klingaman et at., 1992; Kudsk, 1992). Foram relatados resultados de controle satisfatório de plantas daninhas com herbicidas de pós-emergência aplicados em doses de apenas $25 \%$ daquelas especifi cadas no rótulo quando as aplicações ocorreram no início do desenvolvimento das ervas, sob condições favoráveis ao seu crescimento e quando foi usada calibração precisa do pulverizador (Baldwin \& Oliver, 1985; Baldwin et a!., 1988). Além disso, a pesquisa sobre interferência de plantas daninhas em soja estabeleceu que é especialmente importante o controle das mesmas no início da estação de crescimento. Os rendimentos de grãos em geral não são afetados quando as lavouras são mantidas livres de infestações durante as primeiras 3 a 4 semanas, após a semeadura (Barrentine, 1974; Eaton et a!., 1976; Hagood et a!., 1980).

Por outro lado, muitas pesquisas indicam que para controlar ervas de maior tamanho são necessárias doses de herbicidas mais elevadas, o que pode reduzir sua eficiência, representar aumento no custo de produção ou não solucionar o problema das plantas daninhas (Mengarda \& Fleck, 1989; Pinto \& Fleck, 1990; Devlin et al., 1991). Igualmente, doses reduzidas não atuam com eficiência, quando condições de clima seco atrasam a emergência das plantas daninhas (DeFelice et a!., 1989). Desse modo, a desvantagem da aplicação inicial no caso de condições desfavoráveis, ou quando ocorrerem germinações posteriores, é de que sejam necessárias aplicações seqüenciais de doses reduzidas para alcançar o controle satisfatório (DeFelice et a!., 1989; Kudsk, 1992). De toda forma, o agricultor deve assumir o risco do controle inadequado, quando os herbicidas são aplicados em doses menores que as recomendadas (Griffin et al., 1992). 
O principal objetivo desta pesquisa foi investigar a possibilidade de uso de doses reduzidas de herbicidas de pós-emergência com ação graminicida para controlar infestação de papuã em soja com menor quantidade de agroquímicos e reduzir os custos de produção, de modo a colocar a soja em posição mais vantajosa e competitiva para comercialização.

\section{MATERIAL E MÉTODOS}

Durante o ano agrícola 1992/93 foram conduzidos dois experimentos em condições de campo na Estação Experimental Agronômica da UFRS, no município de Eldorado do Sul, RS, empregando-se a cultivar de soja "BR-4" como reagente. Foram instalados em solo Podzólico VermelhoEscuro, distrófico, unidade de mapeamento São Jerônimo, classificado como de textura areno-franco-argilosa, contendo $32 \%$ de argila e 2,2\% de matéria orgânica.

Análise química do solo amostrado um mês antes da semeadura mostrou os seguintes resultados: $\mathrm{pH}$ 5,2 (SMP 6,0); P 17 ppm; K 166 ppm; CTC 6,1 me/dl. Sete meses antes da semeadura da cultura foi procedida aplicação e incorporação de 2 t/ha de calcário. Seis meses antes da semeadura foram aplicados $300 \mathrm{~kg} / \mathrm{ha}$ da fórmula de adubo 5-20-10, correspondendo a $15 \mathrm{~kg} / \mathrm{ha}$ de $\mathrm{N}, 60 \mathrm{~kg} / \mathrm{ha}$ de $\mathrm{P}_{2} \mathrm{O}_{5}$ e 30 $\mathrm{kg} / \mathrm{ha}$ de $\mathrm{K}_{2} 0.0$ preparo do solo foi convencional, através de uma aração realizada 6 meses antes da instalação dos ensaios e de duas gradagens executadas, respectivamente, com antecipação de 20 dias e de 1 dia à instalação dos ensaios.

A semeadura de ambos os experimentos ocorreu no dia 5 de novembro de 1992. A emergência verificou-se após 7 dias, e a colheita foi procedida em 15 de abril de 1993, ou sejam, 154 dias após a emergência da cultura. A semeadura foi realizada com uma semeadeira de impulsão manual, em linhas afastadas de $0,5 \mathrm{~m}$ entre si e à profundidade média de $4 \mathrm{~cm}$. A população média de soja emergida na área experimental foi de 12 plantas $/ \mathrm{m}$ de fila, ou $240 \mathrm{mil}$ plantas/ha. Durante o ciclo da cultura foi necessário realizar aplicações de inseticidas (clorpirifós ou methamidophos) para manter sob controle os insetos-praga que ocorreram.

A área experimental esteve fortemente infestada por papuã (Brachiaria plantaginea), gramínea ocorrente nas parcelas testemunhas em populações iniciais médias de 467 e 525 plantas $/ \mathrm{m}^{2}$, nos experimentos 1 e 2 , respectivamente. Para controlar esta espécie foram testados os herbicidas haloxyfop \{ácido 2-[4-[[3-cloro-5-(trifluorometil)-2-piridinil]oxi]fenóxi]- propanóico $\}$ e sethoxydim \{2-[1-(etoxi imino)butil]-5-[2-(etiltio)- propil]-3-hidróxi-2-ciclohexeno1ona\}, aplicados em pós-emergência. Cada um foi avaliado em cinco tratamentos. Os tratamentos químicos aplicados, suas doses e épocas de utilização constam da Tabela 1 . As doses de $120 \mathrm{~g} /$ ha de haloxyfop e de $184 \mathrm{~g} / \mathrm{ha}$ de sethoxydim foram consideradas como usuais ou plenas.

No experimento 1 , os herbicidas foram pulverizados 7 dias após a semeadura da cultura, quando as plantas de soja estavam emergindo do solo e as de papuã encontravam-se com até uma a duas folhas, exceto os tratamentos com as doses plenas dos herbicidas, que foram aplicadas 21 dias após a semeadura da soja, com plantas de papuã de até dois afilhos. Já no experimento 2, os herbicidas foram aplicados 14 dias após a semeadura da soja, ocasião em que suas plantas apresentavam-se no estádio do primeiro par de folhas simples e as de papuã achavam-se com até três a quatro folhas, exceto os tratamentos com as doses plenas dos herbicidas, aplicados 28 dias após a semeadura, com plantas de papuã de até cinco a seis afilhos. Foram acrescidos dois controles aos tratamentos químicos: testemunha capinada manualmente e testemunha infestada por papuã durante todo ciclo da cultura. Ambos os experimentos receberam três operações de capinas manuais: para o experimento 1, estas foram realizadas aos 21,35 e 56 dias após a data de semeadura, enquanto para o experimento 2 foram realizadas aos 28,42 e 56 dias após a semeadura da soja.

A pulverização dos herbicidas foi realizada em cobertura total da área das parcelas, utilizando-se pulverizador costal de precisão, sendo a pressão mantida constante em $160 \mathrm{kPa}$. Na barra foram usados quatro bicos tipo leque, série 11004 , espaçados em $0,5 \mathrm{~m}$ entre si. O volume de calda herbicida utilizado foi de 250 1/ha. Todas as aplicações de herbicidas ficaram compreendidas no horário entre 9 e 10 horas. Não houve necessidade de aplicar medidas de controle contra outras espécies daninhas na área, como ervas dicotiledôneas, porque ocorreram de modo muito esparso, não interferindo com o objetivo da pesquisa.

As condições meteorológicas nos dias das aplicações dos herbicidas no experimento 1 foram as seguintes: na primeira data de aspersão - temperaturas mínima, máxima e média de $19,0,31,2$ e $25,1{ }^{\circ} \mathrm{C}$, e umidade relativa de $65 \%$; e na segunda data - temperaturas mínima, máxima e média de $19,0,27,0$ e $21,6^{\circ} \mathrm{C}$ e umidade relativa de $70 \%$. Quanto à ocor rência de precipitações pluviais, houve chuvas totalizando $66 \mathrm{~mm}$ dentre os 10 dias precedentes à primeira aplicação e $30 \mathrm{~mm}$ aos 3 dias após a mesma; enquanto na segunda aplicação houve apenas queda de $1 \mathrm{~mm} 5$ dias antes, e o total de 44,2 $\mathrm{mm}$ aos 3 e 4 dias após a mesma.

Quanto ao experimento 2, as condições meteorológicas foram: na primeira data de aplicação - temperaturas mínima, máxima e média de $12,0,27,6$ e $19,8{ }^{\circ} \mathrm{C}$, respectivamente, e umidade relativa do ar de $61 \%$; e na segunda data temperaturas mínima, máxima e média de 13,0 , 25,8 e 19,0 ${ }^{\circ} \mathrm{C}$, e umidade relativa de $65 \%$. Quanto às precipitações pluviais, ocorreram $57,4 \mathrm{~mm}$ dentre os 10 dias precedentes à primeira data e $27,6 \mathrm{~mm}$ nos 10 dias consecutivos; já na segunda data de aplicação houve 44,2 $\mathrm{mm}$ dentre os 10 dias precedentes às pulverizações e 31,7 nos 10 dias consecutivos.

Para comparar os tratamentos foi utilizado o delineamento experimental de blocos ao acaso, com quatro repetições. O tamanho das parcelas foi de $2,5 \times 6,0 \mathrm{~m}$, sendo constituídas por quatro fileiras de soja. A área útil correspondente à avaliação da produtividade da soja foi de 1,0 x 5,0 m, englobando as duas fileiras centrais, desprezando-se $0,5 \mathrm{~m}$ em suas extremidades.

O grau de controle de papuã foi avaliado visualmente em três ocasiões, utilizando-se escala percentual que procurou aferir a fitomassa da gramínea infestante em relação aos padrões. Ao final do ciclo da soja foi obtido o rendimento de grãos de soja, o qual foi referido a $12 \%$ de umidade. 
TABELA 1 - Tratamentos herbicidas testados nos experimentos de controle de papuã em soja com doses reduzidas de compostos com ação graminicida em pós-emergência, EEA/UFRS, Eldorado do Sul, RS, 1992/93.

\begin{tabular}{|c|c|c|c|c|c|}
\hline \multicolumn{4}{|c|}{ Compostos herbicidas $^{1}$} & \multirow{2}{*}{\multicolumn{2}{|c|}{$\frac{\text { Épocas de aplicação }^{2}}{\text { Experimentos }}$}} \\
\hline \multirow{2}{*}{ Nome comum } & \multirow{2}{*}{ Dose (g/ha) } & \multirow{2}{*}{ Nome comercial } & \multirow{2}{*}{ Dose (ml/ha) } & & \\
\hline & & & & 1 & 2 \\
\hline Haloxyfop ${ }^{3}$ & 120 & Verdict & 500 & 3 semanas & 4 semanas \\
\hline Haloxyfop & 90 & Verdict & 375 & 1 semana & 2 semanas \\
\hline Haloxyfop & 60 & Verdict & 250 & 1 semana & 2 semanas \\
\hline Haloxyfop & 30 & Verdict & 125 & 1 semana & 2 semanas \\
\hline $\begin{array}{c}\text { Haloxyfop } \\
+\end{array}$ & 30 & $\begin{array}{c}\text { Verdict } \\
+\end{array}$ & 125 & $\begin{array}{c}1 \text { semana } \\
\mathrm{e}\end{array}$ & $\begin{array}{c}2 \text { semanas } \\
\mathrm{e}\end{array}$ \\
\hline Haloxyfop & 30 & Verdict & 125 & 3 semanas & 4 semanas \\
\hline Sethoxydim ${ }^{4}$ & 184 & Poast & 1000 & 3 semanas & 4 semanas \\
\hline Sethoxydim & 138 & Poast & 750 & 1 semana & 2 semanas \\
\hline Sethoxydim & 92 & Poast & 500 & 1 semana & 2 semanas \\
\hline Sethoxydim & 46 & Poast & 250 & 1 semana & 2 semanas \\
\hline $\begin{array}{c}\text { Sethoxydim } \\
+\end{array}$ & 46 & $\begin{array}{c}\text { Poast } \\
+\end{array}$ & 250 & $\begin{array}{c}1 \text { semana } \\
\mathrm{e}\end{array}$ & $\begin{array}{c}2 \text { semanas } \\
\mathrm{e}\end{array}$ \\
\hline Sethoxydim & 46 & Poast & 250 & 3 semanas & 4 semanas \\
\hline
\end{tabular}

${ }^{1}$ Ao herbicida haloxifop (Verdict) foi adicionado adjuvante Joint na concentraçáo de $0,5 \%$; ao herbicida setoxidim (Poast) foi adicionado adjuvante Assist na dose de 1,5 L/ha;

2 Referidas em semanas após a data de semeadura da cultura de soja.

3 Produto comercial apresentado como concentrado emulsionável contendo concentração de $240 \mathrm{~g} / \mathrm{l}$ de equivalente ácido.

4 Produto comercial apresentado como concentrado emulsionável contendo concentraçáo de $184 \mathrm{~g} / 1$ de ingrediente ativo.

Os dados das variáveis avaliadas foram submetidos à análise da variância pelo teste $\mathrm{F}$, e as médias dos tratamentos foram comparadas através do teste de Duncan. Ambas as análises adotaram nível de significância de $5 \%$ de probabilidade. Para efetuar análise de variância dos dados provenientes das avaliações visuais de controle de papuã, os valores foram previamente transformados em raiz quadrada. Também foram testados os modelos linear e quadrático de regressão para expressar a relação entre produtividade da soja e níveis de controle de papuã.

\section{RESULTADOS E DISCUSSÃO}

Como resultados, constatou-se que, no experimento 1, haloxyfop usado na dose plena alcançou controle praticamente completo de papuã ( $98 \%$ ), enquanto que a dose plena de sethoxydim também propiciou grau satisfatório de controle (88\%) (Tabela 2). Todos os demais tratamentos com herbicidas utilizando doses reduzidas forneceram graus de controle muito baixos, inaceitáveis na prática, exceto aplicação de haloxyfop em doses fracionadas de $30+30 \mathrm{~g} / \mathrm{ha}$, cuja eliminação de papuã situou-se entre 70 e $75 \%$.

No experimento 1 , níveis de produtividade de soja equivalentes aos do tratamento capinado só foram obtidos com doses plenas de haloxyfop e sethoxydim (Tabela 2). A produtividade obtida nas demais aplicações, usando doses reduzidas, foram inferiores àquelas e não diferiram da teste- munha infestada. Verificou-se redução de $58 \%$ na produtividade da soja, quando se comparou a testemunha infestada com a capinada, ou decréscimo de $61 \%$, quando a testemunha infestada foi comparada com a média dos dois melhores tratamentos herbicidas (Tabela 2). Da mesma forma, quando comparado o rendimento do melhor tratamento com doses reduzidas, haloxyfop a $30+30 \mathrm{~g} / \mathrm{ha}$, que proporcionou até $75 \%$ de controle, contra a média dos dois tratamentos de maior produção, constatou-se redução de quase $40 \% \mathrm{em}$ decorrência da interferência do papuã.

No experimento 2, constatou-se que os graus de controle de papuã propiciados pelas doses plenas de haloxyfop e sethoxydim foram equivalentes aos níveis alcançados no experimento $1,98 \%$ e $88 \%$, respectivamente (Tabela 3 ). As demais doses reduzidas de sethoxydim foram insuficientes para propiciar controle satisfatório da gramínea. A dose de $138 \mathrm{~g} / \mathrm{ha}$ atingiu grau de controle de apenas 67\%, em média. No entanto, haloxyfop nas doses reduzidas de 90 e $60 \mathrm{~g} / \mathrm{ha}$ alcançou níveis de controle de papuã ao redor de 80 e $75 \%$, respectivamente. Já o uso deste composto em doses fracionadas de $30+30 \mathrm{~g} / \mathrm{ha}$ proporcionou controle de papuã da ordem de $95 \%$.

Atribui-se a diferença de controle entre experimentos, pelo menos parcialmente, ao fato de que no experimento 1 as plantas de papuã se encontravam em pleno processo de emergência, enquanto no experimento 2 a grande maioria já havia emergido, quando ocorreram as pulverizações de herbicidas. Além disso, considera-se que no primeiro caso as 
TABELA 2 - Controle de papuã em soja com doses reduzidas de herbicidas graminicidas de pós-emergência, experimento 1, EEA/UFRS, Eldorado do Sul, RS, 1992/93.

\begin{tabular}{|c|c|c|c|c|c|}
\hline \multirow{2}{*}{$\begin{array}{c}\text { Tratamentos } \\
\text { testados }\end{array}$} & \multirow{2}{*}{ Dose $(\mathrm{g} / \mathrm{ha})$} & \multicolumn{3}{|c|}{ Avaliaçöes de controle de papuã ${ }^{2}$} & \multirow{2}{*}{$\begin{array}{l}\text { Rendimento de } \\
\text { gräos de soja } \\
(\mathrm{kg} / \mathrm{ha})\end{array}$} \\
\hline & & $\mathbf{I}^{3}$ & II & III & \\
\hline 1. Haloxyfop & 120 & $85,0 \mathrm{ab}^{7}$ & 98,5 a & 98,5 a & 1869 a \\
\hline 2. Haloxyfop & 90 & $50,0 \quad \mathrm{c}$ & $33,7 \mathrm{~cd}$ & $28,7 \mathrm{~b}$ & 917 bc \\
\hline 3. Haloxyfop & 60 & $31,2 \quad d$ & $22,5 \mathrm{de}$ & 16,2 bc & $943 \mathrm{bc}$ \\
\hline 4. Haloxyfop & 30 & 15,1 & 6,3 & $11,2 \quad \mathrm{c}$ & 812 bc \\
\hline 5. Haloxyfop & $2 \times 30$ & $71,2 \mathrm{bc}$ & $70,0 \quad$ b & $75,0 \mathrm{a}$ & $1160 \mathrm{~b}$ \\
\hline 6. Sethoxydim & 184 & $86,2 \mathrm{ab}$ & $82,7 \mathrm{ab}$ & 88,7 a & 1936 a \\
\hline 7. Sethoxydim & 138 & 13,8 & 10,1 & $11,3 \mathrm{~cd}$ & $999 \mathrm{bc}$ \\
\hline 8. Sethoxydim & 92 & 18,7 & 17,5 & $13,8 \quad \mathrm{c}$ & $923 \mathrm{bc}$ \\
\hline 9. Sethoxydim & 46 & 7,6 & 5,1 & $5,1 \mathrm{~cd}$ & $485 \mathrm{c}$ \\
\hline 10. Sethoxydim & $2 \times 46$ & $58,7 \quad \mathrm{c}$ & \multirow{2}{*}{$37,5 \quad$ c } & 29,0 b & 968 bc \\
\hline \multicolumn{2}{|c|}{ 11. Testemunha capinada ${ }^{5}$} & 100,0 a & & $100,0 \mathrm{a}$ & 1757 a \\
\hline \multicolumn{2}{|c|}{ 12. Testemunha infestada ${ }^{6}$} & 0 & 0 & 0 & $741 \mathrm{bc}$ \\
\hline \multicolumn{2}{|c|}{ Coeficiente de variaçẩo (\%) } & 12,5 & 10,7 & 13,2 & 35,7 \\
\hline
\end{tabular}

1 Tratamentos $\mathrm{n}^{2} 2,3,4,7,8$ e 9 aplicados 1 semana após semeadura da soja; tratamentos $\mathrm{n}^{0} 1$ e 6 aplicados 3 semanas após semeadura da soja; tratamentos $n^{2} 5$ e 10, fracionados, foram aplicados parte 1 semana e parte 3 semanas após semeadura da cultura.

2 Utilizada escala percentual para avaliação visual, em que nota zero correspondeu a nenhum controle e nota cem a controle total do papuá. Os dados foram transformados pela raiz quadrada para efetuar a análise da variância.

${ }^{3}$ Avaliaçöes I, II e III realizadas, respectivamente, 3, 5 e 7 semanas após aplicaçăo dos tratamentos $\mathrm{n}^{2} 2,3,4,7,8$ e 9 e 1, 3 e 5 semanas após aplicaçáo do: tratamentos $\mathrm{n}^{\circ} 1,5,6 \mathrm{e} 10$.

4 Corrigido para teor de umidade de $12 \%$.

5 Realizadas três operaçōes de capinas manuais 3, 5 e 8 semanas após semeadura da soja.

${ }_{7}^{6}$ Mantida sem controle da população natural de papuã, ocorrente inicialmente numa densidade média de 467 plantas/m ${ }^{2}$.

7 Médias comparadas nas colunas, seguidas da mesma letra, năo diferem entre si pelo teste de Duncan a $5 \%$ de probabilidade.

plantas encontravam-se numa fase inicial de crescimento, decorrendo daí que nesta situação sua área foliar era mais reduzida para interceptação de herbicida que a das plantas em estádio mais avançado de crescimento, como ocorreu no segundo experimento.

Considere-se ainda que as plantas de soja cobriram as entrelinhas mais tarde no experimento 1 em comparação ao 2 , relativamente à época em que os produtos foram aplicados em cada um destes ensaios. $\mathrm{O}$ uso de doses reduzidas ou não de herbicidas de pós-emergência alcança maior sucesso quando a soja é cultivada em fileiras mais próximas, o que aumenta o nível complementar de controle das ervas e o rendimento da cultura (DeFelice et al., 1989; Peters et al., 1965; Wax \& Pendleton, 1968). Nos experimentos aqui realizados a população de soja foi baixa, cerca de $40 \%$ menor do que a ideal, o que pode ter contribuído para uma emergência mais prolongada e interferência mais intensa do papuã. Esta menor população de soja pode também ter apresentado efeito positivo ao suportar melhor o acamamento que normalmente uma elevada população de papuã ocasiona ao final do ciclo da cultura.

Embora investigando basicamente a ação de herbicidas sobre espécies daninhas dicotiledôneas, diversos autores (Mengarda \& fleck, 1989; DeFelice et al., 1989; Steckel et al., 1990; Devlin et al., 1991; Carey et al., 1992; King \& Oliver, 1992; Klingaman et al., 1992) enfatizam que o uso de doses reduzidas de herbicidas de pós-emergência deve ocor rer cedo e sobre ervas de pequeno tamanho e em crescimento ativo, a fim de obter-se controle satisfatório. Além disso, a prática de doses reduzidas deve ocorrer sob condições ambientes favoráveis (Barrentine, 1989; DeFelice et al., 1989; Griffin et al., 1992 Devlin et al., 1991). Outra constatação é a de que aplicações seqüenciais de doses reduzidas aspergidas cedo sobre ervas extremamente pequenas geralmente as controlam em níveis equivalentes aos de uma única aplicação de doses elevadas usuais, pulverizadas sobre plantas mais desenvolvidas (DeFelice et al., 1989; Steckel et al., 1990; Devlin et al., 1991).

$\mathrm{O}$ efeito alcançado pelo uso de doses reduzidas fracionadas em aplicações sequienciais foi bastante satisfatório, em especial para haloxyfop. A estratégia de aplicações de herbicidas divididas tem sido comprovada em outras situações. Ela tem provido controle mais satisfatório de ervas e permitido redução na quantidade total de herbicida utilizado (Kudsk, 1992). Adicionalmente, em geral ocorre também maior tolerância da cultura em decorrência de aspersões divididas que por efeito de uma única aplicação (Kudsk, 1992). Do mesmo modo como foi verificado por Prostko \& Meade (1993), os resultados aqui relatados comprovaram também que os tratamentos usando metade das doses usuais não foram tão eficientes quanto doses plenas ou aplicações seqüenciais de doses correspondentes a apenas $25 \%$ das recomendadas. Carey et al. (1992) obtiveram também controle satisfatório de capim-arroz em estádio inicial com uso 
TABELA 3 - Controle de papuã em soja com doses reduzidas de herbicidas graminicidas de pós-emergência, experimento 2, EEA/UFRS, Eldorado do Sul, RS, 1992/93.

\begin{tabular}{|c|c|c|c|c|c|c|c|}
\hline \multirow{2}{*}{$\begin{array}{c}\text { Tratamentos } \\
\text { testados }^{1}\end{array}$} & \multirow{2}{*}{ Dose $(\mathrm{g} / \mathrm{ha})$} & \multicolumn{5}{|c|}{ Avaliaçöes de controle de papuä ${ }^{2}$} & \multirow{2}{*}{$\begin{array}{c}\text { Rendimento de } \\
\text { grãos de soja } \\
(\mathrm{kg} / \mathrm{ha})\end{array}$} \\
\hline & & $\mathbf{I}^{3}$ & II & & III & & \\
\hline 1. Haloxyfop & 120 & $87,5 a b c^{7}$ & \multicolumn{2}{|l|}{98,5 a } & \multicolumn{2}{|l|}{98,5 a } & $2692 \mathrm{ab}$ \\
\hline 2. Haloxyfop & 90 & 82,0 bc & \multicolumn{2}{|c|}{78,7 b } & \multicolumn{2}{|c|}{83,2 ab } & $2265 \mathrm{abc}$ \\
\hline 3. Haloxyfop & 60 & $85,7 \mathrm{abc}$ & \multicolumn{2}{|c|}{76,0 bc } & \multicolumn{2}{|c|}{75,7 bc } & $2076 \mathrm{~cd}$ \\
\hline 4. Haloxyfop & 30 & $63,7 \quad d$ & 62,5 & d & 53,7 & d & $1543 \mathrm{de}$ \\
\hline 5. Haloxyfop & $2 \times 30$ & $93,7 \mathrm{ab}$ & \multicolumn{2}{|l|}{$95,0 \mathrm{a}$} & \multicolumn{2}{|l|}{96,2 a } & 2235 bc \\
\hline 6. Sethoxydim & 184 & 81,2 bc & \multicolumn{2}{|l|}{93,7 a } & \multicolumn{2}{|c|}{$87,5 \mathrm{ab}$} & 2819 a \\
\hline 7. Sethoxydim & 138 & 72,5 cd & 66,2 & cd & 62,5 & cd & 1992 cde \\
\hline 8. Sethoxydim & 92 & 46,2 & 35,0 & e & 37,5 & c & 1467 \\
\hline 9. Sethoxydim & 46 & 5,1 & 11,2 & f & 9,0 & f & 939 \\
\hline 10. Sethoxydim & $2 \times 46$ & 40,0 & 33,7 & $\mathrm{e}$ & 35,5 & $\mathrm{e}$ & 1665 \\
\hline \multicolumn{2}{|c|}{ 11. Testemunha capinada ${ }^{5}$} & 100,0 a & \multicolumn{2}{|l|}{100,0 a } & \multicolumn{2}{|l|}{$100,0 \mathrm{a}$} & $2720 \mathrm{ab}$ \\
\hline \multicolumn{2}{|c|}{ 12. Testemunha infestada ${ }^{6}$} & 0 & 0 & $\mathrm{~g}$ & 0 & $\mathrm{~g}$ & 726 \\
\hline \multicolumn{2}{|c|}{ Coeficiente de variação (\%) } & 6,3 & \multicolumn{2}{|l|}{5,2} & \multicolumn{2}{|l|}{7,1} & 18,6 \\
\hline
\end{tabular}

1 Tratamentos $\mathrm{n}^{\circ} 2,3,4,7,8$ e 9 aplicados 2 semanas após semeadura da soja; tratamentos $\mathrm{n}^{\circ} 1$ e 6 aplicados 4 semanas após semeadura da soja; tratamentos $\mathrm{n}^{2} 5$ e 10 , fracionados, foram aplicados parte 2 semanas e parte 4 semanas após semeadura da cultura.

2 Utilizada escala percentual para avaliaçáo visual, em que nota zero correspondeu a nenhum controle e nota cem a controle total do papuá. Os dados forar. transformados pela raiz quadrada para efetuar a análise da variáncia.

3 Avaliaçóes I, II e III realizadas, respectivamente, 3, 5 e 7 semanas após aplicaçáo dos tratamentos n' 2, 3, 4, 7, 8 e 9 e 1, 3 e 5 semanas após aplicação dos tratamentos $\mathrm{n}^{\circ} 1,5,6$ e 10.

4 Corrigido para teor de umidade de $12 \%$,

5 Realizadas trếs operaçóes de capinas manuais 4,6 e 8 semanas após semeadura da soja.

${ }_{7}^{6}$ Mantida sem controle da populaçáo natural de papuá, ocorrente inicialmente numa densidade média de $525 \mathrm{plantas} / \mathrm{m}^{2}$.

${ }^{7}$ Médias comparadas nas colunas, seguidas da mesma letra, năo diferem entre si pelo teste de Duncan a $5 \%$ de probabilidade.

de doses reduzidas em aplicações seqüenciais dos herbicidas graminicidas clethodim e fenoxaprop.

Não pode ser desconsiderada a relação entre dose herbicida e estádio de crescimento das gramíneas. Pesquisas que utilizaram compostos graminicidas de pós-emergência em espécies como milhã, capim pé-de-galinha e setária mostraram que o controle das mesmas foi mais eficiente quando os indivíduos encontravam-se em estádio de crescimento variável de plantas recém-emergidas até início de afilhamento. A partir deste a eficácia de controle diminuiu semivelmente (Chernicky et al., 1984; Deer et al., 1985; Pinto \& Fleck, 1990). Estas constatações explicam os elevados graus de controle de papuã obtidos nos tratamentos padrões com doses usuais, mas aplicados na fase de afilhamento.

Aplicações repetidas com doses reduzidas costumam produzir controle muito mais satisfatório que uma única aplicação em dose elevada. Isto se deve à toxicidade do herbicida que, quando aplicado em dose elevada, pode destruir ao menos parcialmente o floema, impedindo o transporte do mesmo. Doses elevadas produzem fitotoxicidade excessiva na parte aérea da planta, o que ocasiona translocação reduzida do produto (Garcia Torres \& FernandezQuintanilla, 1991). Considerando a concentração herbicida, há evidência de que, com muitos herbicidas, a dose ou concentração passa por um ótimo. Doses acima do ótimo podem causar rápido dano visível à folhagem (Ashton \& Crafts, 1981).
Certas aplicações de herbicidas reduzem a produção de cera epicuticular sobre o tecido foliar e alteram a absorção do mesmo ou de outros herbicidas (Hess, 1985). A redução na deposição de cera resulta num aumento na absorção herbicida. Pode ser o caso dos herbicidas aqui considerados, que inibem também a síntese de lipídios como modo de ação, podendo predispo-los a incrementar a absorção da segunda aplicação através do pré-tratamento.

Além destas hipóteses relacionadas às plantas, supõe-se também que aplicações seqüenciais, mesmo de doses pequenas, teriam condições de abranger o controle de plantas daninhas durante um período mais prolongado, atuando basicamente sobre duas camadas de plantas com épocas distintas de emergência, desde que aplicadas a plantas em estádio bastante inicial de crescimento. Por outro lado, como estes compostos são referidos como de persistência curta no solo, é provável que no experimento 1 pudesse ocorrer emergência adicional de papuã e reinfestação da área de forma mais intensa do que no segundo caso.

No experimento 2, níveis de produtividade de soja, equivalentes aos do tratamento capinado, foram alcançados nas doses plenas dos dois herbicidas e ainda com haloxyfop a $90 \mathrm{~g} / \mathrm{ha}$ e também com aplicações seqüenciais de $30+30$ $\mathrm{g} /$ ha (Tabela 3). Os demais tratamentos com doses reduzidas não forneceram níveis satisfatórios de produtividade, embora tais tratamentos produzissem rendimentos superiores ao da testemunha infestada, exceto sethoxydim a $46 \mathrm{~g} / \mathrm{ha}$, que 
foi equivalente a esta testemunha. Neste experimento, obteve-se decréscimo de produtividade de soja da ordem de $73 \%$ por efeito da interferência da infestação de papuã, quando se compara a testemunha infestada com a capinada, ou aproximadamente a mesma redução quando comparada testemunha infestada com a média dos dois melhores tratamentos de herbicidas. Por outro lado, quando se compara o rendimento do tratamento com haloxyfop a $60 \mathrm{~g} / \mathrm{ha}$, que forneceu cerca de $75 \%$ de controle de papuã, com a média dos dois tratamentos que alcançaram maiores rendimentos, verifica-se que houve redução de $25 \%$ na produtividade em decorrência da interferência exercida.

Os níveis de produtividade de soja obtidos no experimento 1 foram consideravelmente inferiores aos alcançados no segundo. Em parte esses resultados poderiam ser esperados, considerando que os níveis de controle de papuã foram bem mais satisfatórios no segundo ensaio do que no primeiro. As hipóteses prováveis para tal comportamento diferencial podem ser uma ou mais das relacionadas a seguir. No experimento 1, as aplicações de herbicidas realizaram-se sobre plantas de papuã com apenas uma folha ou ainda em plena emergência do solo, as quais, nesta fase, podem não ter absorvido ou translocado quantidades suficientes dos herbicidas aos locais de ação. Grande parte das doses testadas foram reduzidas e, portanto, insuficientes para propiciar adequada ação residual de solo. Em geral, as condições ambientes seguintes às aplicações foram mais favoráveis para ocasionar mais rápida dissipação dos compostos no experimento 1 do que no 2. Aplicação herbicida muito cedo no ensaio 1 permitiu germinação posterior de mais plantas de papuã, que passaram a interferir mais com a cultura. Por fim, em relação às épocas de aplicação, a soja não cobriu as entrelinhas suficientemente cedo, de modo a impedir novas germinações ou suprimir o crescimento das plantas de papuã, no experimento 1. DeFelice et al. (1989) também constataram que o uso de doses herbicidas reduzidas pode originar maiores populações de ervas no final do ciclo da soja. Enquanto Devlin et al. (1991) enfatizam que herbicidas de pós-emergência, usados em baixas doses, apresentam mínima atividade de solo e normalmente não controlam ervas que emergem após a aplicação.

Na Figura 1, encontram-se expostos, em forma gráfica, os resultados referentes à análise de regressão entre rendimento de grãos de soja e níveis de controle de papuã, os quais mostraram relação linear significativa em ambos os experimentos. No ensaio 1, a equação de regressão ajustouse aos dados em nível muito inferior à do ensaio 2 , fato que também pode ser conferido pelos coeficientes de variação para rendimento de grãos (Tabelas 2 e 3). O gráfico indica que elevadas produtividades de soja podem ser alcançadas somente quando se obtém eliminação praticamente completa da gramínea. A equação de regressão encontrada no experimento 1 estima que se perdem $115 \mathrm{~kg} / \mathrm{ha}$ de grãos de soja para cada redução de $10 \%$ no grau de controle de papuã. Já no experimento 2, a estimativa indica diminuição de 187 $\mathrm{kg} / \mathrm{ha}$ de produtividade para cada redução de $10 \%$ no nível de controle da gramínea. Em parte, esta variação de resultados é reflexo dos diferentes potenciais de rendimentos alcançados em cada ensaio. No entanto, diferentemente destes resultados, pesquisas realizadas sob condições diversas e

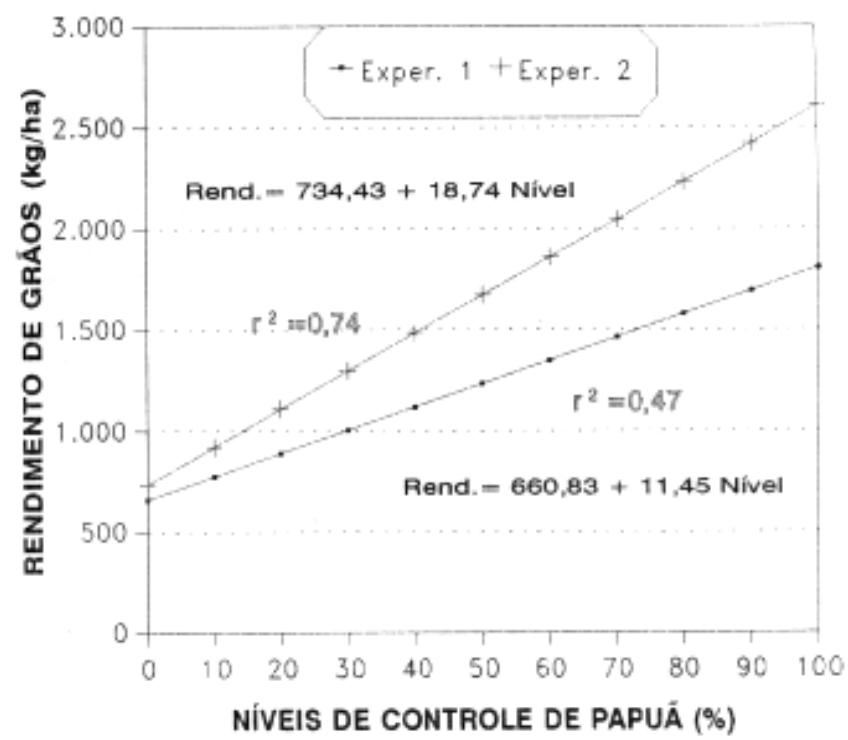

FIG. 1 - Relaçäo entre rendimento de grãos de soja e níveis de controle de papuã, em função da aplicaçáo de herbicidas graminicidas de pós-emergência, para os experimentos 1 e 2, EEA/UFRS, Eldorado do Sul, RS, 1992/93.

incluindo outras espécies daninhas, principalmente dicotiledôneas, mostraram que controle completo de plantas damnhas não é necessário para obter rendimentos máximos de soja (Barrentine, 1974; DeFelice et al., 1989; Hagood et al., 1980; McWorther \& Anderson, 1976). Contudo, o papuã tem mostrado ser uma espécie de grande agressividade vegetativa e de elevada habilidade competitiva, o que também exerce interferência alelopática em soja (Almeida, 1988).

Em decorrência destes resultados, pode-se concluir que é possível alcançar níveis satisfatórios de controle de papuã com doses de herbicidas reduzidas, as quais dependem do produto, da época e do método de aplicação. E viável o uso de doses de haloxyfop inferiores às usuais. Já sethoxydim mostra pouca viabilidade de ser usado nesta modalidade. Aplicações de herbicidas sobre plantas de papuã com três a quatro folhas são mais satisfatórias que sobre plantas com apenas uma a duas folhas. É possível conseguir controle eficiente de papuã com redução de $50 \%$ da dose usual de haloxyfop, desde que esta seja fracionada em aplicações sequienciais, pulverizadas sobre ervas pequenas. Por fim, a resposta linear da produtividade da soja, ern função do nível de controle de papuã, mostra ser necessário que a infestação seja eliminada em alto grau para se atingir máximo rendimento de grãos.

\section{LITERATURA CITADA}

ALMEID A, F.S. A alelopatia e as plantas. Londrina: IAPAR, $1988.60 \mathrm{p}$.

ASHTON, F.M.; CRAFTS, A.S. Absorption and translocation of herbicides. In: Mode of action of herbicides. 2.ed., New York: John Wiley, 1981. p. 20-39. 
BALDWIN, F.L.; BOYD, J.W.; GUY, C.B. Recommended chemicals for weed and brush control. Fayetteville: University of Arkansas Cooperative Extension Service,1991. 118 p.(University of Arkansas, Bulletin MP44).

BALDWIN, F.L.; OLIVER, L.R. A reduced rate intensive management soybean weed control program. In: SOUTHERN WEED SCIENCE SOCIETY, 38., 1985, Proceedings. p.487.

BARRENTINE, W.L. Common cocklebur competition in soybeans. Weed Science, v.22, p.600-602, 1974.

BARRENTINE, W.L. Minimum effective rate of chlorimuron and imazaquin applied to common cocklebur (Xanthium strumarium). Weed Technology, v.3, p.126-130, 1989.

CAREY, V.F.; SMITH JR., R.J.; TALBERT, R.E. Reduced and standard herbicide rates for grass control in rice (Oryza saliva). Weed Technology, v.6, p.409-414, 1992.

CHERNICKY, J.P.; GOSSETT, B.J.; MURPHY, T.R. Factors influencing control of annual grasses with sethoxydim or RO13-8895. Weed Science, v.32, p.174-177, 1984.

DEER, J.F.; MONACO, T.J.; SHEETS, T.J. Response of three annual grasses to fluazifop. Weed Science, v33, p.693-697, 1985.

DEFELICE, M.S.; BROWN, W.B.; ALDRICH, R.J.; SIMS, B.D.; JUDY, D.T.; GUETHLE, D.R. Weed control in soybeans (Glycine max) with reduced rates of postemergence herbicides. Weed Science, v.37, p.365-374, 1989.

DEVLIN, D.L.; LONG, J.H.; MADDUX, L.D. Using reduced rates of postemergence herbicides in soybeans (Glycine max). Weed Technology, v.5, p.834-840, 1991.

EATON, B.J.; RUSS, O.G.; FELTNER, K.C. Competition of velvetleaf, prickly sida, and venice mallow in soybeans. Weed Science, v.24, p.224-228, 1976.

GARCIA TORRES, L.; FERNANDEZ-QUINT ANILLA, C. Comportamiento de los herbicidas en la planta. In: Fundamentos sobre malas hierbas y he rb ici da s. Madrid: Mundi-Prensa, 1991. p.129-157.

GRIFFIN, J.L.; REYNOLDS, D.B.; VIDRINE, P.R.; SAXTON, A.M. Common cocklebur (Xanthium strumarium) control with reduced rates of soil and foliarapplied imazaquin. Weed Technology, v.6, p.847$851,1992$.
HAGOOD JR., E.S.; BAUMAN, T.T.; WILLIAMS JR., J.L.; SCHREIBER, M.M. Growth analys is of soybeans (Glycine max) in competition with velvetleaf (Abutilon theophrasti). Weed Science, v.28, p.729734, 1980.

HESS, F.D. Herbicide absorption and translocation and their relationship to plant tolerances and susceptibility. In: DUKE, S.O., ed., Weed phy siology. Boca Raton: CRC, 1985. v.2, p.191-214.

KING, C.A.; OLIVER, L.R. Application rate and timing of acifluorfen, bentazon, chlorimuron, and imazaquin. Weed Technology, v.6, p.526-534, 1992.

KLINGMAN, T.E.; KING, C.A.; OLIVER, L.R. Effect of application rate, weed species, and weed stage of growth on imazethapyr activity. Weed Science, v.40, p.227-232, 1992.

KUDSK, P. Split decisions help weed control in peas. Shell Agriculture, 13: 29-30, 1992.

McWHORTER, C.G.; ANDERSON, J.M. Bentazon applied postemergence for economical control of common cocklebur in soybeans. Weed Science, v.24, p.391396, 1976.

MENGARDA, I.P.; FLECK, N.G. Atividade herbicida de compostos difeniléteres aplicados em pós-emergência à cultura da soja. Pesquisa Agropecuária Brasileira, v.24, p.531-541, 1989.

PEIERS, E.J.; GEBHARDT, M.R.; STRITZKE, J.F. Interrelations of row spacings, cultivations, and herbicides for weed control in soybeans. Weeds, v.13, p.285-289, 1965.

PINTO, J.J.O;; FLECK, N.G. Comportamento de herbicidas utilizados em pós-emergência no controle de plantas daninhas gramíneas em soja. Pesquisa Agropecuária Brasileira, v.25, p.815-831, 1990.

PROSTKO, E.P.; MEADE, J.A. Reduced rates of postemergence herbicides in conventional soybeans (Glycine max). Weed Technology, v.7, p.365-369, 1993.

STECKEL, L.E.; DEFELICE, M.S.; SIMS, B.D. Integrating reduced rates of postemergence herbicides and cultivatíon for broadleaf weed control in soybeans (Glycine max). Weed Science, v.38, p.541-545, 1990.

WALLACE, R.W.; BELLINDER, R.R. Low-rate applications of herbicides in conventional and reduced tillage potatoes (Solanum tuberosum). Weed Technology, v.4, p.509-513, 1990.

WAX, L.M.; PENDLETON, J.W. Effect of row spacing on weed control in soybeans. Weed Science, v.16, p.462-465, 1968. 